Research Article

\title{
Stabilization of a Class of Nonlinear Underactuated Robotic Systems through Nonsingular Fast Terminal Sliding Mode Control
}

\author{
Yaobin Song, ${ }^{1}$ Hui Li ${ }^{1},{ }^{1,2}$ and Xiaoling Shi ${ }^{1}$ \\ ${ }^{1}$ Department of Mining Engineering, Lvliang University, Lvliang 033001, China \\ ${ }^{2}$ School of Mechanical Engineering, North University of China, Taiyuan 030051, China \\ Correspondence should be addressed to Hui Li; lihui@llhc.edu.cn
}

Received 21 June 2020; Revised 24 October 2020; Accepted 15 November 2020; Published 25 November 2020

Academic Editor: Rafał Stanisławski

Copyright (c) 2020 Yaobin Song et al. This is an open access article distributed under the Creative Commons Attribution License, which permits unrestricted use, distribution, and reproduction in any medium, provided the original work is properly cited.

To facilitate the stabilization of nonlinear underactuated robotic systems under perturbation, a novel nonsingular fast terminal sliding mode control method is proposed. Based on the system transformation into an integrator chain, the combination of twisting-like algorithm and a nonsingular fast terminal sliding mode control technique is employed to achieve the stabilization of the studied systems, which can drive the robot states (joint positions and velocities) to the desired region and then maintain the system at the equilibrium point in finite time. The robustness of the proposed method is validated by the Lyapunov direct method. Finally, numerical simulation results further demonstrate that the proposed method has better performance on the convergent speed of the system state (robot joint positions and velocities) than state-of-the-art methods, especially for the underactuated joints.

\section{Introduction}

Robotics technologies have been widely applied to various industrial applications, such as agriculture $[1,2]$, mining [3], and manufacturing [4-6]. To enable the robots to successfully operate in these fields, the design methodology of robot control is a necessary issue that practitioners need to consider. Among various robot designs, the control of underactuated robots has become an important and attractive research issue [7-9]. Such kind of robots has many advantages, such as low costs, compactness of design, and lightweight. Meanwhile, the difficulties of controller design are also accompanied due to the existence of the robot joints that cannot be driven directly [9]. Besides, the underactuated robots are often highly nonlinear in their dynamics, making the control even challenging when facing uncertainties [10].

To overcome these control difficulties, multiple studies on the control of underactuated robotic systems have been proposed, such as fuzzy control [11-14], optimal control [15-17], partial feedback linearization [18, 19], energy-based approach [20-22], and sliding mode control [23-27]. In particular, the sliding mode control has been a popular control method due to its variable structure controller design that can endow nonlinear systems with good robustness under the uncertainties which are commonly met in real plants [28].

For the control of practical underactuated systems, many researchers have put in lots of efforts [29-31]. One of the most classical systems is the cart-pole system (CPS), which has been taken as the benchmark for control in many studies [32-35]. Based on the opinion of [36], although many researchers have proposed various control schemes for dealing with the upward swing problem [37-39], the control issue for the CPS that has the initial pose of the pendulum at upper-half plan has not been well solved. In addition, the introduction of a nonlinear damping perturbation on the underactuated joint will further worsen the system stability at the equilibrium point, making the control more difficult [40]. Therefore, many studies do not consider such a perturbation. Nevertheless, some studies show that fast terminal sliding mode control can effectively cope with such an issue $[41,42]$.

Inspired by the aforementioned issue, this work attempts to propose a control method that can not only manage the 
CPS under the damping perturbation but also achieve better performance on the convergent speed of the system state. The proposed method firstly requires a coordinate transform to govern a new integration system in accordance with the concept of differential parametrization. Next, a sliding mode control method based on a reaching controller (PD) and a variation of twisting-like controller [43] is proposed, where the sliding mode variables are designed based on the inspiration of the nonsingular fast terminal sliding mode control method [44]. There are two sliding mode variables designed in this control method: one can drive the underactuated joint to the desired position and velocity, while another one can stabilize the overall system state. The stability analysis is also provided via the Lyapunov direct method for proving that the proposed controller can enable the closed-loop system to converge in finite time. Finally, a comparative study is made with state-of-the-art methods based on numerical simulations for demonstrating that the proposed method has better performance on the settling time (convergent speed) of system state.

The contributions of this work are illustrated as follows:

(1) This work uses a method that combines the coordinate transformation and twisting-like methods to model a CPS system, making the dynamics of the CPS system simpler to be controlled

(2) A nonsingular terminal sliding-mode control method is proposed on the simplified underactuated robotic system, where the robustness of the designed method is proved via the Lyapunov method

(3) The simulation results show that the proposed method has better control performance on the convergent speed, than other state-of-the-art methods

The remainder of this paper is organized as follows: Section 2 describes the problem statement of this work; Section 3 illustrates the detailed methodologies on the system treatments and controller design, as well as the stability analysis; Section 4 shows the comparative study based on numerical simulations; Section 5 briefly summarizes this paper.

\section{Problem Statement}

Consider the following dynamics of an $n$-link underactuated robotic system:

$$
\mathbf{M}(\mathbf{q}) \ddot{q}+\mathbf{C}(\mathbf{q}, \dot{q}) \dot{q}=\tau_{g}(\mathbf{q})+\tau_{d}(\mathbf{q})+\mathbf{B} \tau_{c},
$$

where $\mathbf{q} \in \mathbb{R}^{n}$ refers to the robot joint position; $\mathbf{M}(\mathbf{q}) \in \mathbb{R}^{n \times n}$ refers to the inertia matrix (symmetric positive-definite); $\mathbf{C}(\mathbf{q}, \dot{q})$ refers to the Coriolis matrix; $\tau_{g}(\mathbf{q}) \in \mathbb{R}^{n}$ refers to the torques generated from the gravity; $\tau_{d}(\mathbf{q}) \in \mathbb{R}^{n}$ refers to the torques generated from the viscous friction; $\tau_{c} \in \mathbb{R}^{n}$ refers to the control inputs; and $\mathbf{B} \in \mathbb{R}^{n \times n}$ refers to a coefficient matrix.

Let the initial robot joint positions and velocities be $\mathbf{q}_{0} \in \mathbb{R}^{n}$ and $\dot{q}_{0} \in \mathbb{R}^{n}$, respectively, and the robot joint positions and velocities be $\mathbf{q}_{d} \in \mathbb{R}^{n}$ and $\dot{q}_{d} \in \mathbb{R}^{n}$, respectively.
The objective of the designed controller $\tau_{c}$ is to drive the studied robotic system from the initial state $\left(\mathbf{q}_{0}\right.$ and $\left.\dot{q}_{0}\right)$ to the goal state $\left(\mathbf{q}_{d}\right.$ and $\left.\dot{q}_{d}\right)$ in finite time. Note that the robot states are known and all parameters in (1) are determined and the goal state is selected as an unstable equilibrium point.

Remark 1. The torques generated from the viscous friction $\tau_{d}(\mathbf{q})$ are nonlinear disturbances and only apply to the underactuated joints, making the stability of the studied system to be easily destroyed.

\section{Methodologies}

This section provides the detailed methodologies on the design of the nonsingular fast terminal sliding mode control. Besides, the corresponding stability is proved through the Lyapunov direct method. The overall schematic diagram is illustrated in Figure 1.

3.1. System Transformation. This studied underactuated robotic system has the following parameters that satisfy the aforementioned nonlinear model (1):

$$
\begin{aligned}
\mathbf{q} & =\left[q_{1}, q_{2}\right]^{T} \in \mathbb{R}^{2}, \\
\mathbf{M}(\mathbf{q}) & =\left[\begin{array}{cc}
m_{1}+m_{2} & m_{2} l \cos q_{2} \\
m_{2} l \cos q_{2} & m_{2} l^{2}
\end{array}\right] \in \mathbb{R}^{2 \times 2}, \\
\mathbf{C}(\mathbf{q}, \dot{q}) & =\left[\begin{array}{cc}
0 & -m_{2} l \sin q_{2} \\
0 & 0
\end{array}\right] \in \mathbb{R}^{2 \times 2}, \\
\boldsymbol{\tau}_{g} & =\left[\begin{array}{c}
0 \\
m_{2} g l \sin q_{2}
\end{array}\right] \in \mathbb{R}^{2 \times 2}, \\
\boldsymbol{\tau}_{d} & =\left[\begin{array}{ll}
0 & d
\end{array}\right]^{T} \in \mathbb{R}^{2 \times 2}, \\
\mathbf{B} & =\left[\begin{array}{ll}
1 & 0
\end{array}\right]^{T} \in \mathbb{R}^{2 \times 2}, \\
\boldsymbol{\tau}_{c} & =u \in \mathbb{R} .
\end{aligned}
$$

Define

$$
\begin{aligned}
& \bar{q}_{1}=\frac{q_{1}}{l}, \\
& \bar{q}_{2}=q_{2}, \\
& \bar{u}=\frac{u}{m_{2} g}, \\
& \bar{m}=\frac{m_{1}}{m_{2}} .
\end{aligned}
$$

A normalized system of (2) can be derived:

$$
\left[\begin{array}{cc}
1+\bar{m} & \cos \bar{q}_{2} \\
\cos \bar{q}_{2} & 1
\end{array}\right] \ddot{\bar{q}}+\left[\begin{array}{c}
-\dot{\bar{q}}_{2}^{2} \sin \bar{q}_{2} \\
-\sin \bar{q}_{2}
\end{array}\right]=\left[\begin{array}{c}
\bar{u} \\
-d \dot{\bar{q}}_{2}
\end{array}\right],
$$

where $\bar{q}=\left[\bar{q}_{1}, \bar{q}_{2}\right]^{T} \in \mathbb{R}^{2}$.

To form a closed control loop, let 


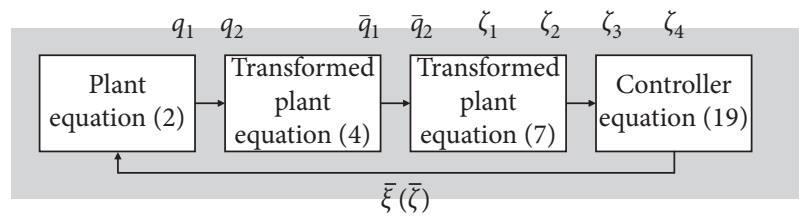

FIGURE 1: Overall schematic diagram of the proposed method.

$\bar{u}=\bar{m} \eta+\eta \sin ^{2} \bar{q}_{2}-\dot{\bar{q}}_{2}^{2} \sin \bar{q}_{2}+\sin \bar{q}_{2} \cos \bar{q}_{2}-d \dot{\bar{q}}_{2} \cos \bar{q}_{2}$.

Then, system (4) becomes

$$
\ddot{\bar{q}}=\left[\begin{array}{c}
-\cos \bar{q}_{2} \\
1
\end{array}\right] \eta+\left[\begin{array}{c}
\sin \bar{q}_{2}-d \dot{\bar{q}}_{2} \\
0
\end{array}\right] .
$$

Next, constructing a new system with new state variables $\zeta=\left[\zeta_{1}, \zeta_{2}, \zeta_{3}, \zeta_{4}\right]^{T} \in \mathbb{R}^{4}$ and a new control input $\xi \in \mathbb{R}$, we obtain

$$
\begin{aligned}
& \dot{\zeta}_{1}=\zeta_{2}, \\
& \dot{\zeta}_{2}=\zeta_{3}+\phi\left(\zeta_{3}, \zeta_{4}\right), \\
& \dot{\zeta}_{3}=\zeta_{4}, \\
& \dot{\zeta}_{4}=\xi
\end{aligned}
$$

First, define two transforms

$$
\begin{aligned}
& \zeta_{1}=\bar{q}_{1}+\ln \left(\varphi\left(\bar{q}_{2}\right)\right), \\
& \zeta_{3}=\tan \bar{q}_{2},
\end{aligned}
$$

where

$$
\varphi\left(\bar{q}_{2}\right)=\frac{1+\tan \left(\bar{q}_{2} / 2\right)}{1-\tan \left(\bar{q}_{2} / 2\right)} .
$$

Then, the corresponding $\zeta_{2}$ and $\zeta_{4}$ can be obtained:

$$
\begin{aligned}
& \zeta_{2}=\dot{\bar{q}}_{1}+\frac{\dot{\bar{q}}_{2}}{\cos \bar{q}_{2}}, \\
& \zeta_{4}=\frac{\dot{\bar{q}}_{2}}{\cos ^{2} \bar{q}_{2}} .
\end{aligned}
$$

Besides, to satisfy (7),

$$
\phi\left(\zeta_{3}, \zeta_{4}\right)=\frac{\zeta_{4}}{\left(1+\zeta_{3}^{2}\right)^{3 / 2}}\left(\zeta_{3} \zeta_{4}-d\left(1+\zeta_{3}^{2}\right)\right)
$$

Remark 2. $\phi\left(\zeta_{3}, \zeta_{4}\right)$ is a nonlinear term to represent the perturbation that causes the plant to be unstable. Besides, with the convergence of the control input $\zeta_{4}$, this term also converges, indicating that the perturbation vanishes in the steady state. For the detailed proof, readers can refer to Appendix.

Based on the concept of the differential parameterizations, introduce a normalized time $\bar{t}$, where $\mathrm{d} \bar{t}=\varepsilon \mathrm{d} t$. Correspondingly, a change of coordinate on (7) is formulated:

$$
\begin{aligned}
& \bar{\zeta}=\operatorname{diag}\left(\varepsilon^{2}, \varepsilon^{1}, \varepsilon^{0}, \varepsilon^{-1}\right) \zeta, \\
& \bar{\xi}=\varepsilon^{-2} \xi,
\end{aligned}
$$

where $\bar{\zeta}=\left[\bar{\zeta}_{1}, \bar{\zeta}_{2}, \bar{\zeta}_{3}, \bar{\zeta}_{4}\right]^{T} \in \mathbb{R}^{4}$ refers to the new state variables under the normalized time $\bar{t} ; \bar{\xi} \in \mathbb{R}$ refers to the new control input under the normalized time; and $\varepsilon=\sqrt{g / l} \in \mathbb{R}^{+}$ refers to the scale of time.

Remark 3. The normalized time $\bar{t}$ is dimensionless, and the differentiation of $\bar{\zeta}$ is thus performed based on the normalized time $\bar{t}$.

Finally, combining (12) which is equivalent to system (2), the plant can be represented by

$$
\dot{\bar{\zeta}}=\Gamma(\bar{\zeta}, \bar{\xi})
$$

where $\Gamma: \mathbb{R}^{4} \times \mathbb{R} \longrightarrow \mathbb{R}^{4}$.

3.2. Controller Design. This section reveals the controller design, and the following definition will be used throughout this paper.

\section{Definition 1.}

$$
\begin{gathered}
\rho^{a}(b)=|b|^{a} \operatorname{sign}(b), \\
\operatorname{sign}(b)= \begin{cases}1, & b>0, \\
0, & b=0, \\
-1, & b<0 .\end{cases}
\end{gathered}
$$

Based on the core idea of sliding mode control, the control input is generated from two modes: the reaching mode and the sliding mode, where the reaching mode drives the state variables to the sliding mode manifold and then the sliding mode keeps the state variables to move along the sliding mode manifold until they converge to the goal state.

Herein, the control law $\bar{\xi}(\bar{\zeta})$ is therefore composed of two controllers:

$$
\bar{\xi}(\bar{\zeta})=\bar{\xi}_{r}(\bar{\zeta})+\bar{\xi}_{s}(\bar{\zeta})
$$

where $\bar{\xi}_{r}(\bar{\zeta})$ refers to the controller for the reaching mode and $\bar{\xi}_{s}(\bar{\zeta})$ refers to the controller for the sliding mode.

The controller for the reaching mode is realized in a PD form:

$$
\bar{\xi}(\bar{\zeta})=-\frac{\lambda_{1} \bar{\zeta}_{3}+\lambda_{2} \bar{\zeta}_{4}}{\lambda_{3}},
$$

where $\lambda_{1}, \lambda_{2}, \lambda_{3} \in \mathbb{R}^{+}$.

The controller for the sliding mode is composed of two sliding mode manifolds $\left(s_{1}=0\right.$ and $\left.s_{2}=0\right)$ :

$$
\bar{\xi}_{s}(\bar{\zeta})=-\frac{\lambda_{4} \operatorname{sign}\left(s_{1}\right)+\lambda_{5} \operatorname{sign}\left(s_{2}\right)}{\lambda_{3}},
$$

where $\lambda_{4}, \lambda_{5} \in \mathbb{R}^{+}$and $\lambda_{4}>\lambda_{5}$; the two sliding mode manifolds are constructed as 


$$
\begin{aligned}
s_{1}= & \lambda_{1} \rho^{v_{1}}\left(\bar{\zeta}_{1}\right)+\left(\lambda_{1}+\lambda_{2}\right) \rho^{v_{1}+v_{2}}\left(\bar{\zeta}_{2}\right) \\
& +\left(\lambda_{2}+\lambda_{3}\right) \rho^{v_{2}+v_{3}}\left(\bar{\zeta}_{3}\right)+\lambda_{3} \rho^{v_{4}}\left(\bar{\zeta}_{4}\right), \\
s_{2}= & \lambda_{1} \rho^{v_{2}+v_{3}}\left(\bar{\zeta}_{2}\right)+\lambda_{2} \rho^{v_{3}+v_{4}}\left(\bar{\zeta}_{3}\right)+\lambda_{3} \rho^{v_{4}}\left(\bar{\zeta}_{4}\right),
\end{aligned}
$$

with $v_{1}, v_{2}, v_{3}, v_{4} \in \mathbb{R}^{+}$.

\subsection{Stability Analysis}

Theorem 1. Consider plant (13) with the following closedloop control law:

$$
\bar{\xi}(\bar{\zeta})=-\frac{\lambda_{1} \bar{\zeta}_{3}+\lambda_{2} \bar{\zeta}_{4}}{\lambda_{3}}+-\frac{\lambda_{4} \operatorname{sign}\left(s_{1}\right)+\lambda_{5} \operatorname{sign}\left(s_{2}\right)}{\lambda_{3}},
$$

where

$$
\begin{aligned}
s_{1}= & \lambda_{1} \rho^{v_{1}}\left(\bar{\zeta}_{1}\right)+\left(\lambda_{1}+\lambda_{2}\right) \rho^{v_{1}+v_{2}}\left(\bar{\zeta}_{2}\right)+\left(\lambda_{2}+\lambda_{3}\right) \rho^{v_{2}+v_{3}}\left(\bar{\zeta}_{3}\right) \\
& +\lambda_{3} \rho^{v_{4}}\left(\bar{\zeta}_{4}\right), \\
s_{2}= & \lambda_{1} \rho^{v_{2}+v_{3}}\left(\bar{\zeta}_{2}\right)+\lambda_{2} \rho^{v_{3}+v_{4}}\left(\bar{\zeta}_{3}\right)+\lambda_{3} \rho^{v_{4}}\left(\bar{\zeta}_{4}\right),
\end{aligned}
$$

with $\quad \lambda_{i} \in \mathbb{R}^{+}(i=1,2,3,4,5), \quad \lambda_{4}>\lambda_{5}, \quad$ and $\quad v_{i} \in \mathbb{R}^{+},(i$ $=1,2,3,4)$, and the two sliding mode variables $\left(s_{1}\right.$ and $\left.s_{2}\right)$ can converge to zero in finite time, making the overall closed-loop system asymptotically stable.

Proof. This proof uses the Lyapunov direct method to prove the stability of the proposed control method. Select a Lyapunov function as

$$
V(\bar{\zeta})=\frac{\lambda_{1}}{2 \lambda_{3}} \bar{\zeta}_{3}^{2}+\frac{1}{2} \bar{\zeta}_{4}^{2}+\frac{1}{2 \lambda_{3} v_{4}} s_{1}^{2}+\frac{1}{2 \lambda_{3} v_{4}} s_{2}^{2}
$$

Then, the derivative of this Lyapunov function is

$$
\begin{aligned}
\dot{V}(\bar{\zeta})= & -\frac{\lambda_{2}}{\lambda_{3}} \bar{\zeta}_{4}^{2} \\
& +\frac{1}{\lambda_{3}} \bar{\zeta}_{4}\left(\lambda_{4} \operatorname{sign}\left(s_{1}\right)+\lambda_{5} \operatorname{sign}\left(s_{2}\right)\right) \\
& +\frac{s_{1} \dot{s}_{1}}{2 \lambda_{2} v_{4}}+\frac{s_{2} \dot{s}_{2}}{2 \lambda_{2} v_{4}} \\
= & -\frac{\lambda_{2}}{\lambda_{3}} \bar{\zeta}_{4}^{2}+\frac{1}{\lambda_{3}} \bar{\zeta}_{4}\left(\lambda_{4} \operatorname{sign}\left(s_{1}\right)+\lambda_{5} \operatorname{sign}\left(s_{2}\right)\right) \\
& +\beta_{1}\left(s_{1}, \dot{s}_{1}\right)+\beta_{2}\left(s_{2}, \dot{s}_{2}\right),
\end{aligned}
$$

where

$$
\begin{aligned}
& \beta_{1}\left(s_{1}, \dot{s}_{1}\right)=-\left|\bar{\zeta}_{4}\right|^{v_{4}-1}\left(\mu_{1} s_{1}^{2}+\mu_{2}\left|s_{1}\right|+\mu_{3} \phi\left(\bar{\zeta}_{3}, \bar{\zeta}_{4}\right)\right), \\
& \beta_{2}\left(s_{2}, \dot{s}_{2}\right)=-\left|\bar{\zeta}_{4}\right|^{v_{4}-1}\left(\mu_{4} s_{2}^{2}+\mu_{5}\left|s_{2}\right|+\mu_{6} \phi\left(\bar{\zeta}_{3}, \bar{\zeta}_{4}\right)\right)
\end{aligned}
$$

with $\mu_{i} \in \mathbb{R}^{+},(i=1,2,3,4,5,6)$.

By Young's inequality, the following inequality can be obtained:

$$
\begin{array}{r}
\frac{1}{\lambda_{3}} \bar{\zeta}_{4}\left(\lambda_{4} \operatorname{sign}\left(s_{1}\right)+\lambda_{5} \operatorname{sign}\left(s_{2}\right)\right) \\
\leq \frac{1}{2 \lambda_{3}}\left(\bar{\zeta}_{4}^{2}+\left(\lambda_{4} \operatorname{sign}\left(s_{1}\right)+\lambda_{5} \operatorname{sign}\left(s_{2}\right)\right)^{2}\right) .
\end{array}
$$

Therefore, an upper bound exists in (22):

$$
\begin{aligned}
\dot{V}(\bar{\zeta}) \leq & -\frac{2 \lambda_{2}-1}{2 \lambda_{3}} \bar{\zeta}_{4}^{2}-\frac{1}{2 \lambda_{3}}\left(\lambda_{4} \operatorname{sign}\left(s_{1}\right)+\lambda_{5} \operatorname{sign}\left(s_{2}\right)\right)^{2} \\
& -\left|\bar{\zeta}_{4}\right|^{v_{4}-1}\left(\mu_{1} s_{1}^{2}+\mu_{2}\left|s_{1}\right|+\mu_{3} \phi\left(\bar{\zeta}_{3}, \bar{\zeta}_{4}\right)\right) \\
& -\left|\bar{\zeta}_{4}\right|^{v_{4}-1}\left(\mu_{4} s_{2}^{2}+\mu_{5}\left|s_{2}\right|+\mu_{6} \phi\left(\bar{\zeta}_{3}, \bar{\zeta}_{4}\right)\right) .
\end{aligned}
$$

Since $\phi\left(\bar{\zeta}_{3}, \bar{\zeta}_{4}\right)$ is bounded (as proved in the Appendix), a conclusion that the overall system is asymptotically stable can be made.

\section{Simulations}

To validate the performance of the proposed controller, a comparative study is made based on numerical simulations in MATLAB. In this comparative study, the control method proposed in [36] is used for the comparison, where it has been shown that the proposed method has better performance than the control method [45]. Therefore, this paper only presents the comparison between the proposed method and the method in [36]. The used parameters for the numerical simulations are depicted in Table 1 . The initial state is selected as $\mathbf{q}_{0}=[-0.5,0.3]^{T}$ and $\dot{q}_{0}=[0.1,-0.2]^{T}$. The goal state is selected as $\mathbf{q}_{d}=[0,0]^{T}$ and $\dot{q}_{d}=[0,0]^{T}$. Note that the used units are radians and radians/second.

Figure 2 and Table 2 present the performances of the proposed control method and the control method in [36]. These results demonstrate that the proposed method has better performance on the settling time, especially for the underactuated joint $q_{1}$.

Figure 3 demonstrates the stability of the designed sliding mode variables $s_{1}$ and $s_{2}$, where these two sliding mode variables converge to zero in finite time. Based on these two sliding mode variables, the designed controller can stabilize the system to the desired state. 
TABle 1: Parameters of the controller.

\begin{tabular}{lc}
\hline Parameters & Values \\
\hline$\varepsilon$ & 0.2 \\
$d$ & 0.8815 \\
$\lambda_{1}$ & 1.5 \\
$\lambda_{2}$ & 8 \\
$\lambda_{3}$ & 4 \\
$\lambda_{4}$ & 6 \\
$\lambda_{5}$ & 0.8 \\
$v_{1}$ & 0.8 \\
$v_{2}$ & 0.2 \\
$v_{3}$ & 0.8 \\
$v_{4}$ & 1 \\
\hline
\end{tabular}
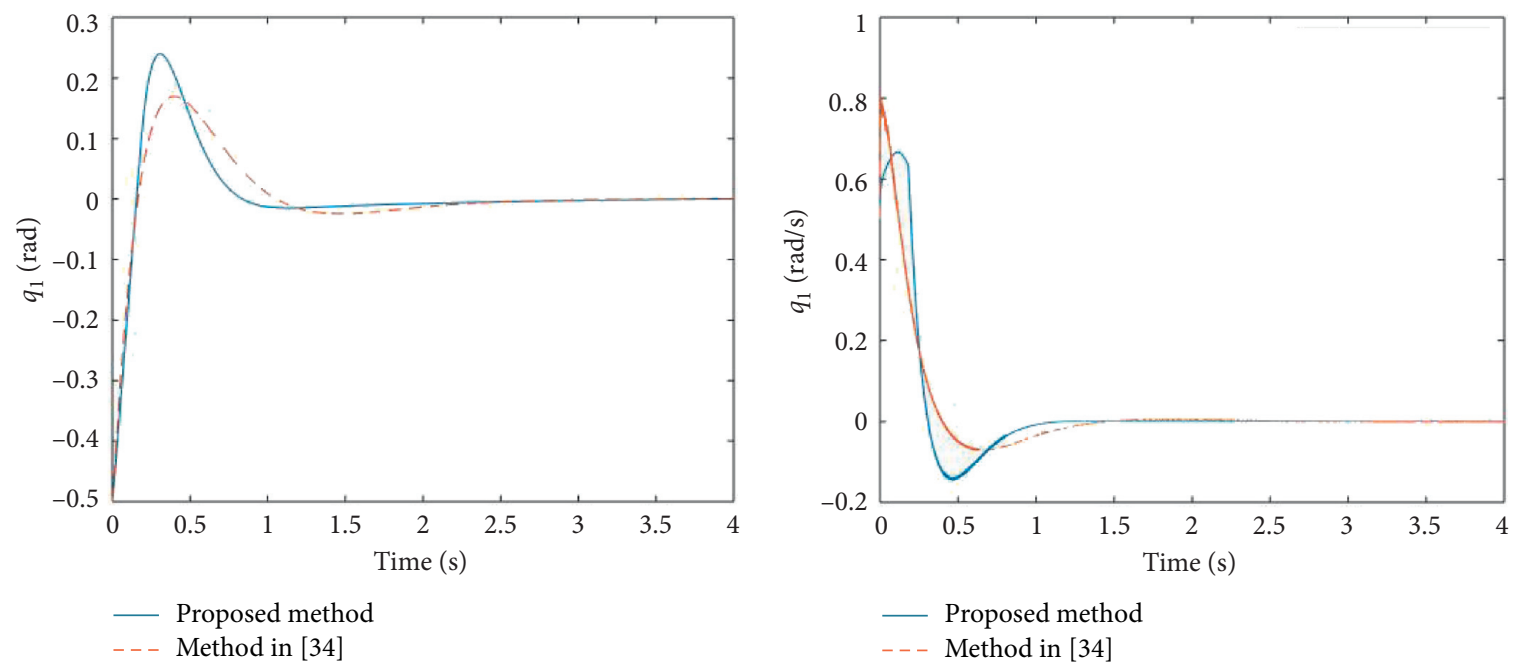

(a)

(b)

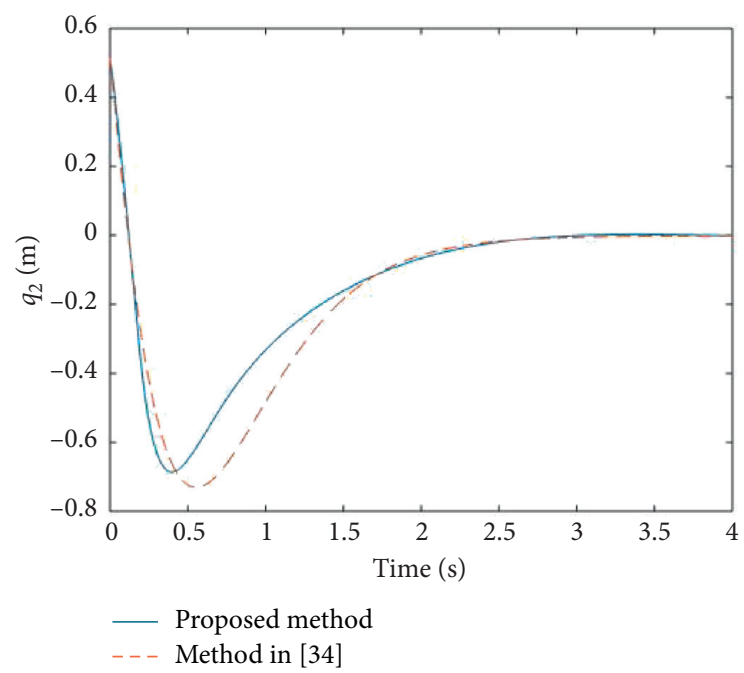

(c)

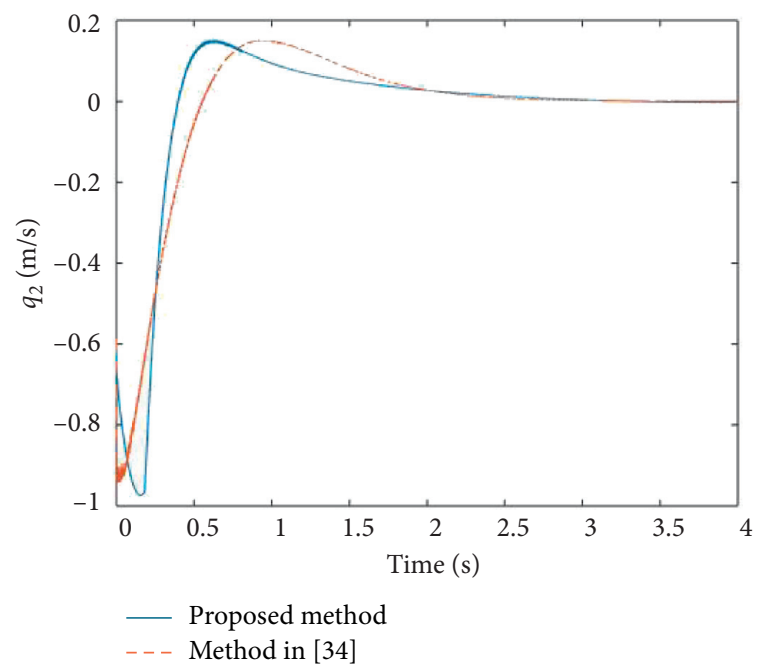

(d)

FIgURe 2: Performance of system state under the proposed method and the method in [36].

Table 2: Performance of settling time.

\begin{tabular}{|c|c|c|c|c|}
\hline & \multicolumn{4}{|c|}{ Settling time (s) } \\
\hline & $q_{1}$ & $\dot{q}_{1}$ & $q_{2}$ & $\dot{q}_{2}$ \\
\hline Proposed method & 1.6534 & 0.9457 & 2.5997 & 2.2618 \\
\hline Method in [36] & 2.1412 & 1.1878 & 2.5640 & 2.1761 \\
\hline
\end{tabular}




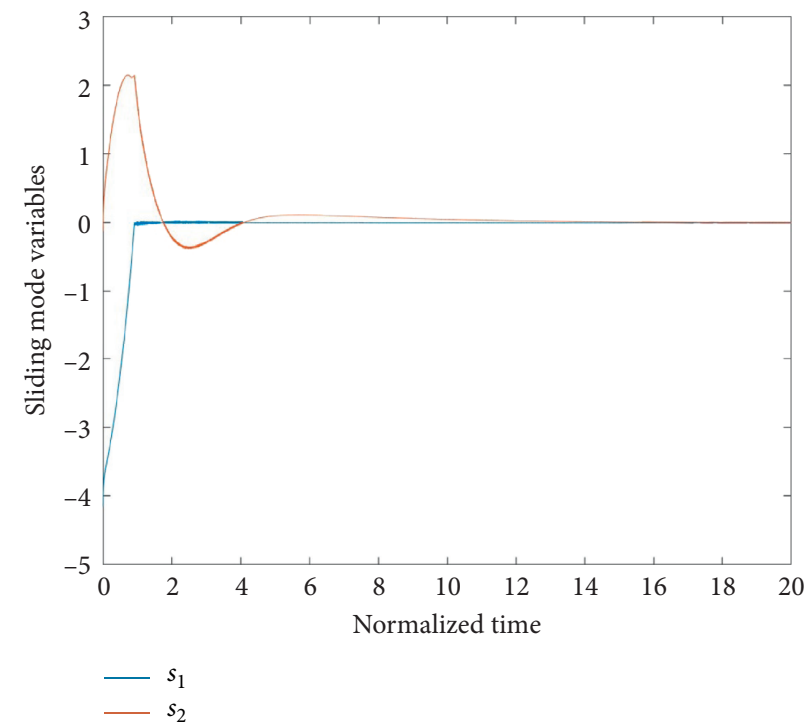

FIGURE 3: Convergence of sliding mode variables.

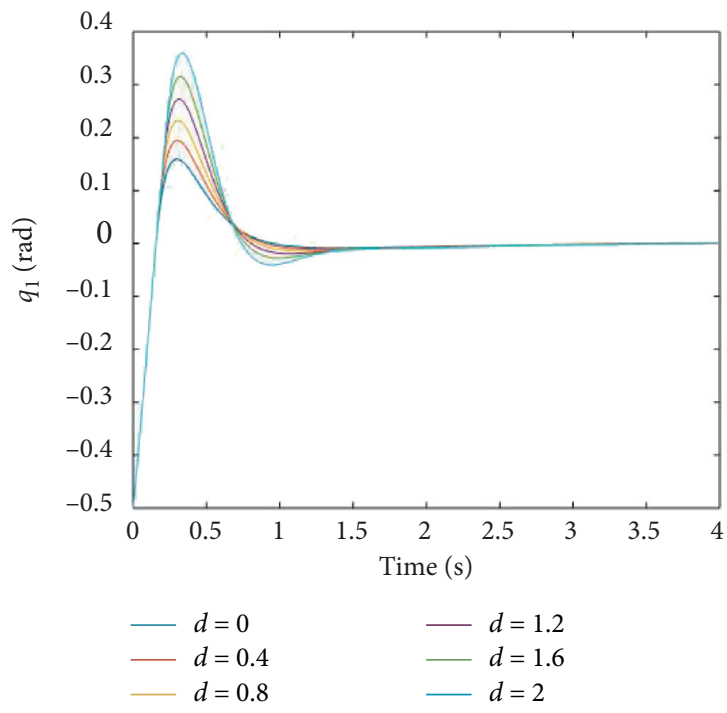

(a)

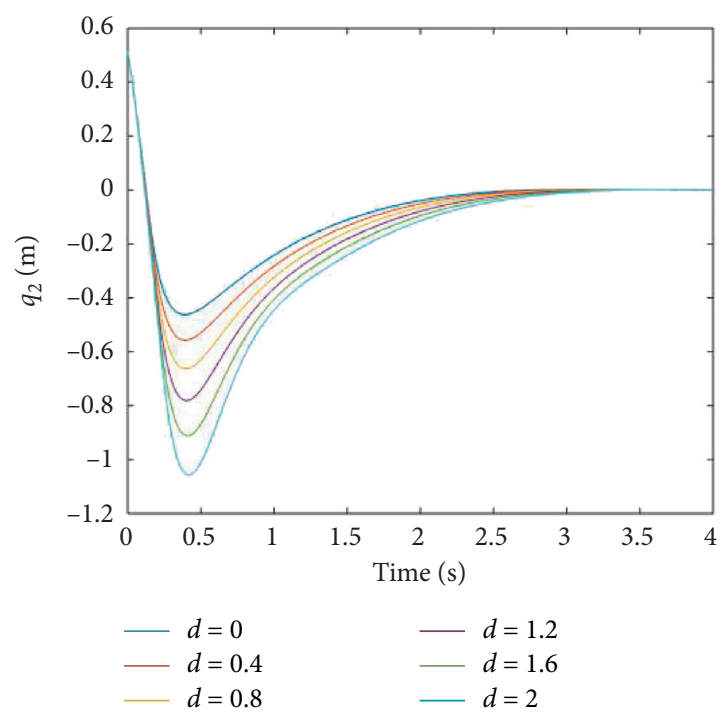

(c)

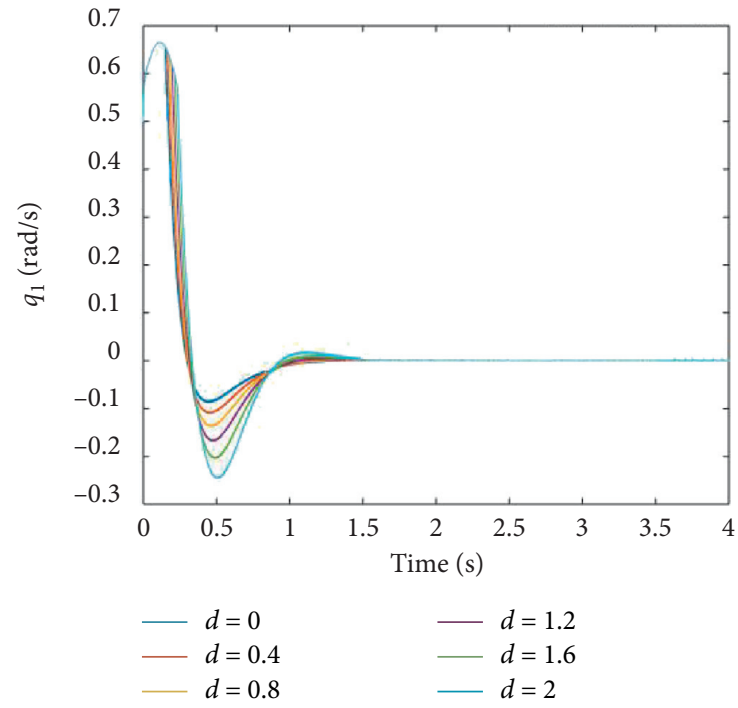

(b)

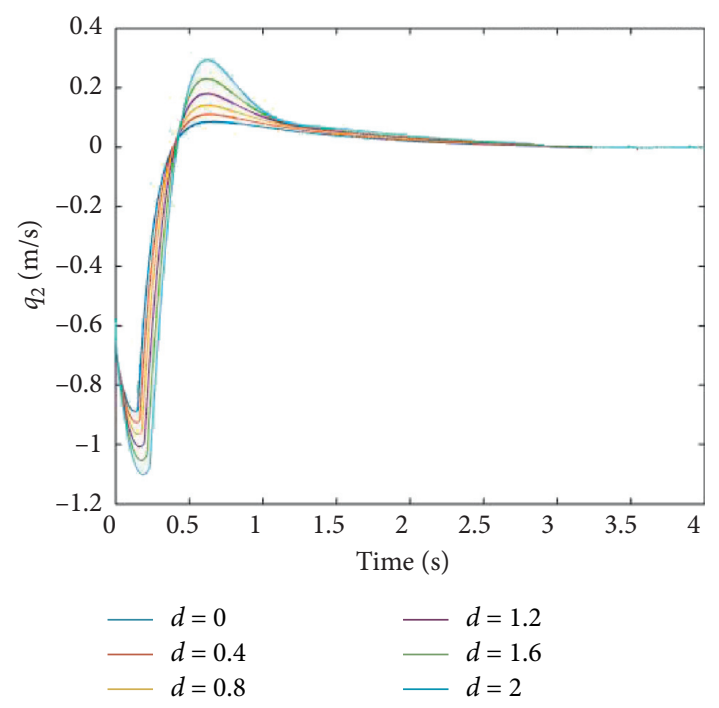

(d)

FIgURE 4: Performance of the proposed method under different damping perturbations. 
To validate the robustness of the proposed control method, the testing of the studied robotic system under different damping perturbations is conducted. Herein, $d$ is changed from 0 to 2, as shown in Figure 4. The results show that the system control becomes difficult with the increase of the damping perturbation $d$. Nevertheless, the proposed control method can manage such perturbation within a range.

\section{Conclusions}

This paper presents a novel control method for controlling a class of nonlinear underactuated robotic systems under nonlinear damping perturbation. The devised method is based on the concept of nonsingular fast terminal sliding mode controls which can obviously improve the settling time (response time). Especially for the underactuated robot joints, the method can not only reduce the settling time but also achieve stability. In addition, this control method is robust to a range of damping perturbations and can ensure finite-time convergence. The simulation results show that the studied underactuated system under the proposed method has better performance on the settling time (or convergent speed) than the state-of-the-art methods and validate that the proposed method can adapt to various damping perturbations. In the future, the trajectory tracking problem under such a perturbation will be an interesting direction for further research.

\section{Appendix}

\section{Convergence of Control Input $\zeta_{4}$}

$$
\begin{aligned}
\phi\left(\zeta_{3}, \zeta_{4}\right) & =\frac{\zeta_{4}}{\left(1+\zeta_{3}^{2}\right)^{3 / 2}}\left(\zeta_{3} \zeta_{4}-d\left(1+\zeta_{3}^{2}\right)\right) \\
& =\frac{\zeta_{3} \zeta_{4}^{2}}{\left(1+\zeta_{3}^{2}\right)^{3 / 2}}-\frac{d \zeta_{4}}{\left(1+\zeta_{3}^{2}\right)^{1 / 2}}
\end{aligned}
$$

Let

$$
\begin{aligned}
& \phi_{1}\left(\zeta_{3}\right)=\frac{\zeta_{3}}{\left(1+\zeta_{3}^{2}\right)^{3 / 2}} \\
& \phi_{2}\left(\zeta_{3}\right)=\frac{1}{\left(1+\zeta_{3}^{2}\right)^{1 / 2}}
\end{aligned}
$$

$\phi_{1}\left(\zeta_{3}\right)$ and $\phi_{2}\left(\zeta_{3}\right)$ satisfy

$$
\begin{aligned}
& \left|\phi_{1}\left(\zeta_{3}\right)\right| \leq \frac{2 \sqrt{3}}{9}, \\
& \left|\phi_{2}\left(\zeta_{3}\right)\right| \leq 1
\end{aligned}
$$

Therefore, the following inequality holds

$$
\left|\phi\left(\zeta_{3}, \zeta_{4}\right)\right| \leq \frac{2 \sqrt{3}}{9} \zeta_{4}^{2}-d \zeta_{4},
$$

indicating that the nonlinear perturbation converges to zero when $\zeta_{4}$ converges to zero.

\section{Data Availability}

All data generated during the study are included within the article.

\section{Conflicts of Interest}

The authors declare that there are no conflicts of interest.

\section{Acknowledgments}

The authors would like to thank Lvliang Key Laboratory of Maintenance and Inspection in Coal Mine Mechanical Equipment. This work was supported under Scientific and Technological Achievements Programs of Higher Education Institutions in Shanxi (TSTAP) (No. 2020CG050) and Science and Technology Project of Lvliang City in 2019 (pressure relief and permeability improvement technology by integrated hydraulic flushing and cutting for low permeability coal seam containing methane) (No. GXZDYF2019080).

\section{References}

[1] D. C. Slaughter, D. K. Giles, and D. Downey, "Autonomous robotic weed control systems: a review," Computers and Electronics in Agriculture, vol. 61, no. 1, pp. 63-78, 2008.

[2] L. J. Kutz, G. E. Miles, P. A. Hammer et al., "Robotic transplanting of bedding plants," Transactions of the ASAE, vol. 30, no. 3, pp. 586-590, 1987.

[3] K. Kato and S. Hirose, "Development of the quadruped walking robot, TITAN-IX-mechanical design concept and application for the humanitarian de-mining robot," Advanced Robotics, vol. 15, no. 2, pp. 191-204, 2001.

[4] C. Y. Weng, W. C. Tan, and I. M. Chen, "A survey of dual-arm robotic issues on assembly tasks," in ROMANSY 22-Robot Design, Dynamics and Control, pp. 474-480, Springer, Berlin, Germany, 2019.

[5] C.-Y. Weng, Q. Yuan, F. Suarez-Ruiz, and I.-M. Chen, "A telemanipulation-based human-robot collaboration method to teach aerospace masking skills," IEEE Transactions on Industrial Informatics, vol. 16, no. 5, pp. 3076-3084, 2020.

[6] A. Cherubini, R. Passama, A. Crosnier, A. Lasnier, and P. Fraisse, "Collaborative manufacturing with physical human-robot interaction," Robotics and Computer-Integrated Manufacturing, vol. 40, pp. 1-13, 2016.

[7] L. Birglen, T. Laliberté, and C. M. Gosselin, Underactuated Robotic Hands, Springer, Berlin, Germany, 2007.

[8] M. W. Spong, "Underactuated mechanical systems," in Control Problems in Robotics and Automation, pp. 135-150, Springer, Berlin, Germany, 1998.

[9] W. Chen, Y. Q. Yu, and X. P. Zhang, "A survey on the underactuated robots," Machine Design and Research, vol. 21, no. 4, pp. 22-25, 2005. 
[10] H. Wang, Y. Pan, S. Li, and H. Yu, "Robust sliding mode control for robots driven by compliant actuators," IEEE Transactions on Control Systems Technology, vol. 27, no. 3, pp. 1259-1266, 2018.

[11] O. Begovich, E. N. Sanchez, and M. Maldonado, "TakagiSugeno fuzzy scheme for real-time trajectory tracking of an underactuated robot," IEEE Transactions on Control Systems Technology, vol. 10, no. 1, pp. 14-20, 2002.

[12] X. J. Ma and Z. Q. Sun, "Output tracking and regulation of nonlinear system based on Takagi-Sugeno fuzzy model," IEEE Transactions on Systems, Man, and Cybernetics, Part B (Cybernetics), vol. 30, no. 1, pp. 47-59, 2000.

[13] H. J. Lee, "Sampled-data observer-based output-feedback fuzzy stabilization of nonlinear systems: exact discrete-time design approach," Fuzzy Sets and Systems, vol. 201, pp. 20-39, 2012.

[14] C. H. Huang, W. J. Wang, and C. H. Chiu, "Design and implementation of fuzzy control on a two-wheel inverted pendulum," IEEE Transactions on Industrial Electronics, vol. 58, no. 7, pp. 2988-3001, 2010.

[15] I. I. Hussein and A. M. Bloch, "Optimal control of underactuated nonholonomic mechanical systems," IEEE Transactions on Automatic Control, vol. 53, no. 3, pp. 668-682, 2008.

[16] S. Kim and S. Kwon, "Nonlinear optimal control design for underactuated two-wheeled inverted pendulum mobile platform," IEEE/ASME Transactions on Mechatronics, vol. 22, no. 6, pp. 2803-2808, 2017.

[17] J. Li, X. Guo, Z. Li, and W. Chen, "Stochastic adaptive optimal control of under-actuated robots using neural networks," Neurocomputing, vol. 142, pp. 190-200, 2014.

[18] M. W. Spong, "Partial feedback linearization of underactuated mechanical systems," in Proceedings of IEEE/RSJ International Conference on Intelligent Robots and Systems IROS'94, pp. 314-321, IEEE, Munich, Germany, 1994.

[19] L. Freidovich, A. Shiriaev, F. Gordillo, F. Gómez-Estern, and J. Aracil, "Partial-energy-shaping control for orbital stabilization of high frequency oscillations of the Furuta pendulum," in Proceedings of the 2007 46th IEEE Conference on Decision and Control, pp. 4637-4642, IEEE, New Orleans, LA, USA, 2007.

[20] I. Duleba and J. Z. Sasiadek, "Non-holonomic motion planning based on Newton algorithm with energy optimization," IEEE Transactions on Control Systems Technology, vol. 11, no. 3, pp. 355-363, 2003.

[21] X. Xin and M. Kaneda, "Analysis of the energy-based control for swinging up two pendulums," IEEE Transactions on Automatic Control, vol. 50, no. 5, pp. 679-684, 2005.

[22] I. Fantoni, R. Lozano, and M. W. Spong, "Energy based control of the pendubot," IEEE Transactions on Automatic Control, vol. 45, no. 4, pp. 725-729, 2000.

[23] D. Liu, J. Yi, D. Zhao, and W. Wang, "Adaptive sliding mode fuzzy control for a two-dimensional overhead crane," Mechatronics, vol. 15, no. 5, pp. 505-522, 2005.

[24] J. X. Xu, Z. Q. Guo, and T. H. Lee, "Design and implementation of integral sliding-mode control on an underactuated two-wheeled mobile robot," IEEE Transactions on Industrial Electronics, vol. 61, no. 7, pp. 3671-3681, 2013.

[25] R. Xu and Ü. Özgüner, "Sliding mode control of a class of underactuated systems," Automatica, vol. 44, no. 1, pp. 233-241, 2008.

[26] H. Ashrafiuon and R. S. Erwin, "Sliding mode control of underactuated multibody systems and its application to shape change control," International Journal of Control, vol. 81, no. 12, pp. 1849-1858, 2008.

[27] S. C. Moon, W. G. Lee, and S. G. Lee, "Adaptive sliding mode control of overhead cranes with varying cable length," Journal of Mechanical Science and Technology, vol. 27, no. 3, pp. 885-893, 2013.

[28] S. Li, M. Zhou, and X. Yu, "Design and implementation of terminal sliding mode control method for PMSM speed regulation system," IEEE Transactions on Industrial Informatics, vol. 9, no. 4, pp. 1879-1891, 2012.

[29] Y. Wu, N. Sun, H. Chen, and Y. Fang, "Adaptive output feedback control for 5-DOF varying-cable-length tower cranes with cargo mass estimation," IEEE Transactions on Industrial Informatics, p. 1, 2020.

[30] T. Yang, N. Sun, H. Chen, and Y. Fang, "Observer-based nonlinear control for tower cranes suffering from uncertain friction and actuator constraints with experimental verification," IEEE Transactions on Industrial Electronics, p. 1, 2020.

[31] H. Chen and N. Sun, "Nonlinear control of underactuated systems subject to both actuated and unactuated state constraints with experimental verification," IEEE Transactions on Industrial Electronics, vol. 67, no. 9, pp. 7702-7714, 2020.

[32] M. H. Abedinnasab, Y. J. Yoon, and M. S. Saeedi-Hosseiny, "High performance fuzzy-Padé controllers: introduction and comparison to fuzzy controllers," Nonlinear Dynamics, vol. 71, no. 1-2, pp. 141-157, 2013.

[33] C. C. Chung and J. Hauser, "Nonlinear control of a swinging pendulum,” Automatica, vol. 31, no. 6, pp. 851-862, 1995.

[34] V. N. Pilipchuk and R. A. Ibrahim, "Dynamics of a twopendulum model with impact interaction and an elastic support," Nonlinear Dynamics, vol. 21, no. 3, pp. 221-247, 2000.

[35] M. E. Semenov, D. V. Shevlyakova, and P. A. Meleshenko, "Inverted pendulum under hysteretic control: stability zones and periodic solutions," Nonlinear Dynamics, vol. 75, no. 1-2, pp. 247-256, 2014.

[36] C. Aguilar-Ibáñez, J. Mendoza-Mendoza, and J. Dávila, "Stabilization of the cart pole system: by sliding mode control," Nonlinear Dynamics, vol. 78, no. 4, pp. 2769-2777, 2014.

[37] J. Á. Acosta, R. Ortega, A. Astolfi, and I. Sarras, "A constructive solution for stabilization via immersion and invariance: the cart and pendulum system," Automatica, vol. 44, no. 9, pp. 2352-2357, 2008.

[38] J. A. Acosta, R. Ortega, A. Astolfi, and A. D. Mahindrakar, "Interconnection and damping assignment passivity-based control of mechanical systems with underactuation degree one," IEEE Transactions on Automatic Control, vol. 50, no. 12, pp. 1936-1955, 2005.

[39] F. Gordillo and J. Aracil, "A new controller for the inverted pendulum on a cart," International Journal of Robust and Nonlinear Control, vol. 18, no. 17, pp. 1607-1621, 2008.

[40] L. Qiao and W. Zhang, "Trajectory tracking control of AUVs via adaptive fast nonsingular integral terminal sliding mode control," IEEE Transactions on Industrial Informatics, vol. 16, no. 2, pp. 1248-1258, 2020.

[41] L. Qiao and W. Zhang, "Adaptive non-singular integral terminal sliding mode tracking control for autonomous underwater vehicles," IET Control Theory \& Applications, vol. 11, no. 8, pp. 1293-1306, 2017.

[42] F. Gómez-Estern and A. J. Van der Schaft, "Physical damping in IDA-PBC controlled underactuated mechanical systems," European Journal of Control, vol. 10, no. 5, pp. 451-468, 2004.

[43] A. Polyakov and A. Poznyak, "Reaching time estimation for "super-twisting" second order sliding mode controller via 
Lyapunov function designing," IEEE Transactions on Automatic Control, vol. 54, no. 8, pp. 1951-1955, 2009.

[44] L. Yang and J. Yang, "Nonsingular fast terminal sliding-mode control for nonlinear dynamical systems," International Journal of Robust and Nonlinear Control, vol. 21, no. 16, pp. 1865-1879, 2011.

[45] S. Riachy, Y. Orlov, T. Floquet, R. Santiesteban, and J. P. Richard, "Second-order sliding mode control of underactuated mechanical systems I: local stabilization with application to an inverted pendulum," International Journal of Robust and Nonlinear Control: IFAC-Affiliated Journal, vol. 18, no. 4-5, pp. 529-543, 2008. 